\title{
ORIGINAL ARTICLE Effects of orthotic therapeutic electrical stimulation in the treatment of patients with paresis associated with acute cervical spinal cord injury: a randomized control trial
}

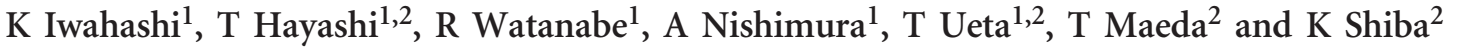

Study design: A randomized controlled trial.

Objectives: To determine the effects of orthotic therapeutic electrical stimulation (TES) on the hand in patients with paresis associated with acute cervical spinal cord injury.

Setting: Spinal Injuries Center, Fukuoka, Japan.

Methods: The study included patients treated for spinal cord injuries (Frankel classification, grades B and C) at our institution within 1 week post injury between May 2011 and December 2014. The patients were allocated randomly to TES and control groups at the time of admission and underwent TES+conventional training or conventional training alone, respectively. Both hands of each patient were treated in the same way. The primary outcome was total passive motion (TPM) of the fingers (degrees). The secondary outcomes were edema $(\mathrm{cm})$ and the upper-extremity motor scores of the International Standards for the Neurological Classification of Spinal Cord Injury (ISNCSCl). After randomization, outcomes were assessed at 1 week, 1 month and 3 months post injury in both groups.

Results: Twenty-nine individuals were assessed at 3 months (15, TES; 14, control). There were no significant between-group differences for TPM of the fingers, edema and upper-extremity motor scores at 1 week, 1 month and 3 months after injury, although TPM of the fingers tended to be lower in the control group.

Conclusions: It is unclear from the results of this study whether TES has a therapeutic effect on TPM, edema or the upper-extremity motor score of the ISNCSCI. The results of this study provide useful data for future meta-analyses.

Spinal Cord (2017) 55, 1066-1070; doi:10.1038/sc.2017.74; published online 27 June 2017

\section{INTRODUCTION}

Motor paralysis due to spinal cord injury causes a considerable reduction or loss of voluntary movements, and when the patient is incapable of appreciable movement and remains immobilized for a long period, contractures often occur. ${ }^{1}$ Contractures are a common and important complication of spinal cord injury, and they may have a considerable influence on the activities of daily living (ADL). Rehabilitation has been performed to prevent and treat articular contractures, and the application of programmed stimulations to the paralyzed limb using functional electric stimulation (FES) through a number of stimulating electrodes has been reported to allow for the re-establishment of targeted movements. ${ }^{2,3}$ In addition, various kinds of devices and methods have been studied regarding the use of FES for treating patients with spinal cord injuries, and the treatment has been reported to be useful. ${ }^{4-6}$

However, randomized control studies using therapeutic electrical stimulation (TES) for patients with cervical spinal cord injury are few, $, 4,78$ and our search of the literature revealed that no randomized control study had been conducted on patients in the acute phase.

In this study, we hypothesized that adding TES training to conventional training starting from the acute phase may prevent contractures of the fingers. Consequently, the purpose of this study was to determine the effects of TES using orthotic TES starting from the acute phase.

\section{PATIENTS AND METHODS}

\section{Patients}

Between May 2011 and December 2014, 134 acute spinal cord injury patients were admitted to Spinal Injuries Center, Fukuoka, Japan within 1 week of injury (110 male, 24 female, mean age: $62.0 \pm 19.2$ years). The classification of these spinal injuries according to the Frankel classification ${ }^{9}$ at 1 week after injury is as follows: grade A comprised 49 patients; grade B, 21; grade C, 22; grade $\mathrm{D}, 17$; and grade $\mathrm{E}, 25$ patients.

This study included patients with paresis associated with acute cervical spinal injuries classified as grades B and C according to the Frankel grading system; grades $\mathrm{A}, \mathrm{D}$ and $\mathrm{E}$ were excluded. In addition, the following comprised exclusion criteria for TES: dementia, mental illness, use of ventilators, and injury to the fingers or forearms. Using a permuted block design for allocation, the patients were randomly divided into the TES group and the control group. The block size of the permuted block design for randomization was set to two. Random allocation, enrollment and assignment were performed by the authors. The random allocation sequence was concealed from the authors until interventions were assigned.

${ }^{1}$ Department of Rehabilitation Medicine, Japan Organization of Occupational Health and Safety, Spinal Injuries Center, Fukuoka, Japan and ${ }^{2}$ Department of Orthopedic Surgery, Japan Organization of Occupational Health and Safety, Spinal Injuries Center, Fukuoka, Japan

Correspondence: Dr T Hayashi, Department of Orthopedic Surgery, Japan Organization of Occupational Health and Safety, Spinal Injuries Center, Igisu 550-4, lizuka city, Fukuoka 820-8508, Japan.

E-mail: tetsuo884hayashi@yahoo.co.jp

Received 30 December 2016; revised 22 April 2017; accepted 22 May 2017; published online 27 June 2017 


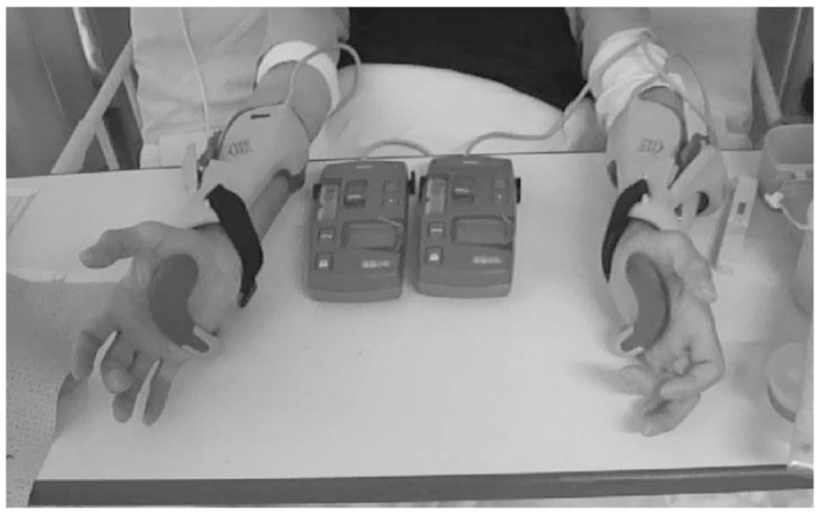

Figure 1 Therapeutic Electrical Stimulation was performed using NESS $\mathrm{H} 200$ hand rehabilitation system. A full colour version of this figure is available at the Spinal Cord journal online.

\section{Intervention}

The TES group received TES in addition to conventional training, whereas, the control group underwent conventional training alone. TES was administered using a neuroprosthesis (NESS H200, Bioness Inc., Valencia, CA; Figure 1). For the TES training, the training time was as follows: 5 min twice daily at 1 week after injury; 10 min twice daily at 2 weeks after injury; 15 min twice daily at 3 weeks after injury; and 20 min twice daily at 4 weeks after injury and thereafter. The intensity of the stimulation was individually adjusted to obtain an optimal motor reaction without any side effects such as pain or skin irritation, and it was usually fixed over time. The electrodes were positioned over the extensor digitorum, extensor pollicis brevis, flexor policis longus and thenar muscles to activate the flexors and extensors of the fingers and thumb. ${ }^{3}$ TESs were performed at patients' bedside or training room for rehabilitation in supine or sitting positions.

Participants were classified by the Zancolli classification systems. ${ }^{10}$ The primary outcome was a total passive motion (TPM) of the fingers. ${ }^{11}$ The secondary outcomes were upper-extremity motor scores of the ISNCSCI ${ }^{12}$ and edema. After random allocation, outcomes were collected at 1 week, 1 month and 3 months after injury in both hands. The anatomic level of the cervical spinal cord injury was assessed by two spinal surgeons using magnetic resonance imaging and computed tomography. TPM of the fingers was measured separately on the right and left sides based on the sum of the flexion-extension range of motion in the metacarpophalangeal (MP), proximal interphalangeal (PIP) and distal interphalangeal (DIP) joints of the first to fifth fingers (14 joints, 28 motions: $1420^{\circ}$ in total). For the edema, the circumferences of the MP joints from the index finger to the little finger were measured. For the upper-extremity motor score, the total score of key muscles in C5 to T1 according to the ISNCSCI was calculated. Blinded assessors were not used.

Our institutional review board approved this research project, and all subjects provided written informed consent before participating in the study. We registered this trial with University hospital Medical Information Network (UMIN) retrospectively according to CONSORT.

\section{Statistical analysis}

The Mann-Whitney $U$-test was used to compare the difference between the TES group and the control groups for each outcome. Multiple comparisons between the TES and control groups were performed at 1 week, 1 month and 3 months after injury using the Turkey-Kramer test; the comparisons were carried out separately for the right and left sides. $P<0.05$ was considered statistically significant.

Statistical analyses were performed using SPSS computer software (version 20, IBM Corp, Armonk, NY, USA). Values were expressed as mean \pm s.d.

\section{RESULTS}

Of the 134 patients admitted to our hospital within 1 week after injury, 43 had Frankel grades B and C spinal function. Of these

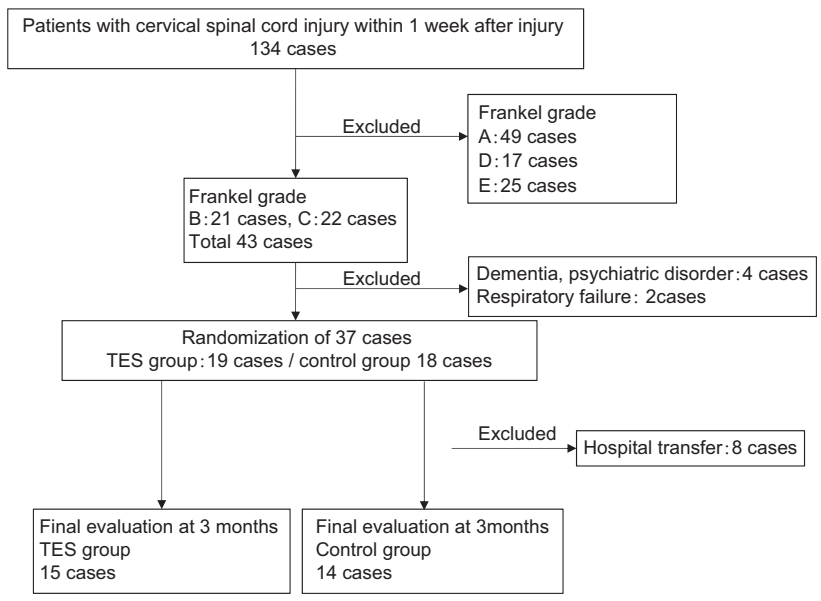

Figure 2 Flow chart of this study.

Table 1 Demographic data at 1 week after injury

\begin{tabular}{lcc}
\hline & TES group & Control group \\
\hline Cases & 15 & 14 \\
Sex(M/F) & $15 / 0$ & $13 / 1$ \\
Age & $57.7 \pm 16.9$ & $59.4 \pm 18.5$ \\
& & \\
TPM of fingers $\left(^{\circ}\right)$ & & \\
Rt & & $1294.6 \pm 131.5$ \\
Lt & $1233.7 \pm 91.7$ & $1374.3 \pm 96.8$ \\
Edema $(\mathrm{cm})$ & $1330.0 \pm 89.3$ & \\
Rt & & $20.1 \pm 1.0$ \\
Lt & $20.6 \pm 0.8$ & $20.1 \pm 1.0$
\end{tabular}

UEMS (/50 points)

Rt $\quad 7.1 \pm 5.1 \quad 6.1 \pm 4.0$

$\begin{array}{lll}\text { Lt } & 6.3 \pm 4.9 & 6.6 \pm 3.8\end{array}$

Abbreviations: TES, therapeutic electrical stimulation; TPM, total passive motion; UEMS, upper extremity motor score.

Values were expressed as mean \pm s.d.

These data were obtained after randomization.

43 patients, 4 with dementia and mental illness and 2 with respiratory failure were excluded, and the remaining 37 were randomly assigned to each group. Eight patients (four patients in the TES group and four patients in the control group) were transferred to other hospitals within 3 months of admission because of an aggravation of their general condition (pneumonia, 3 cases; cerebrovascular disease, 2 cases; Ischemic heart disease, 2 cases; and ileus, 1 case), and, as a result, 29 patients finally underwent measurements for 3 months without any important harms or unintended effects (Figure 2). The TES group was composed of 15 patients (15 male, mean age: $57.7 \pm 16.9$ years) and the control group consisted of 14 patients (13 male and 1 female, mean age: $59.4 \pm 18.5$ years). At 1 week after injury, according to the Frankel classification, 6 patients were grade $\mathrm{B}$ and 9 patients were grade $\mathrm{C}$ in the TES group, whereas, in the control group, 10 patients were grade B and 4 patients were grade C. Regarding level of injury, in the TES group, 7 patients had spinal injuries occurring at C3/4, 4 had injuries at C4/5, 3 at $\mathrm{C} 5 / 6$ and 1 patient at C6/7. In the control group, 6 patients had spinal injuries occurring at C3/4, 1 patient at C4/5 injury, 4 at C5/6, and 3 at C6/7.

The two groups were comparable in terms of age, gender, TPM of the fingers, edema or upper-extremity motor score (Table 1). 
Table 2 Outcome data at baseline, 1 week and 3 months

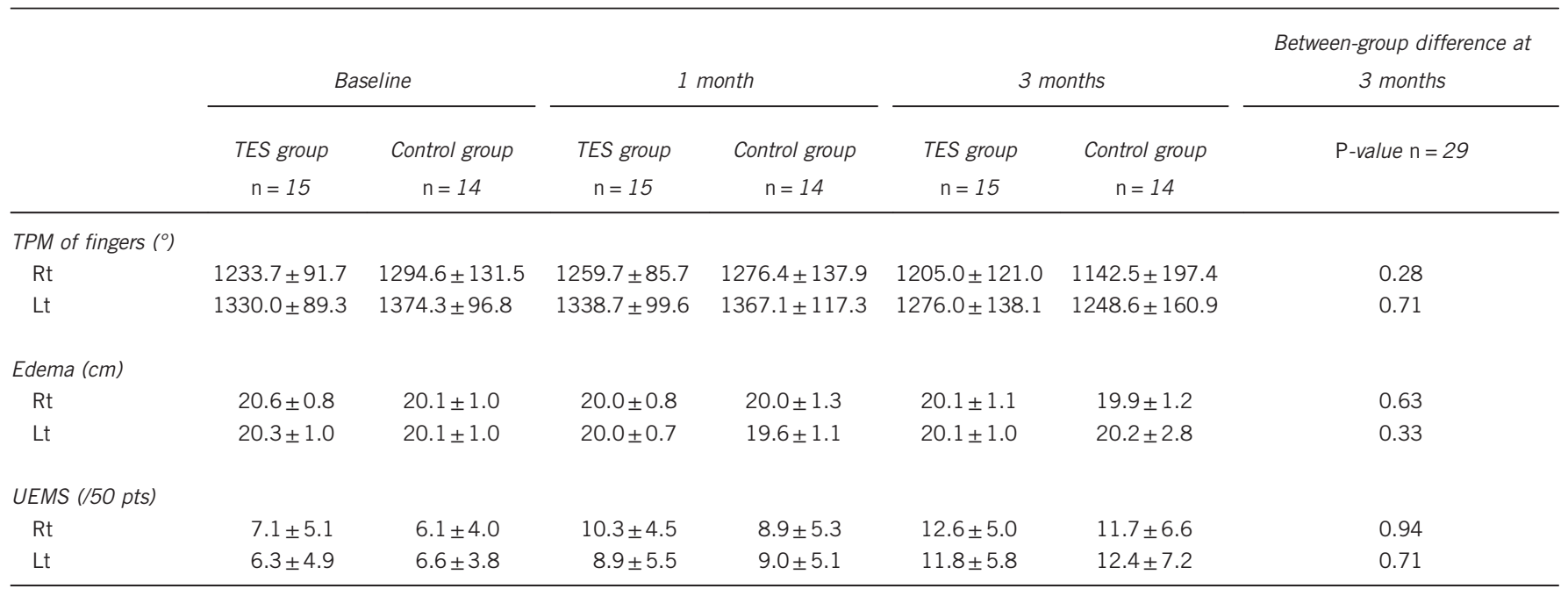

Abbreviations: TES, therapeutic electrical stimulation; TPM, total passive motion; UEMS, upper extremity motor score.

Values were expressed as mean \pm s.d.

Mann-Whitney U-test was used to compare the difference between TES group and the control group.

a

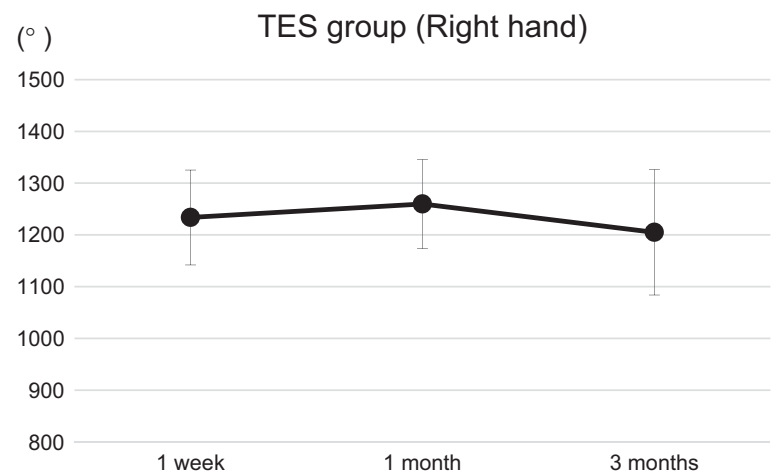

C

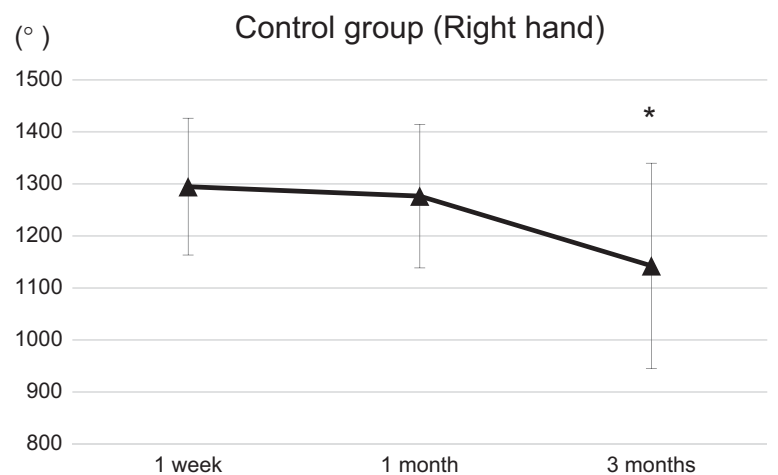

b

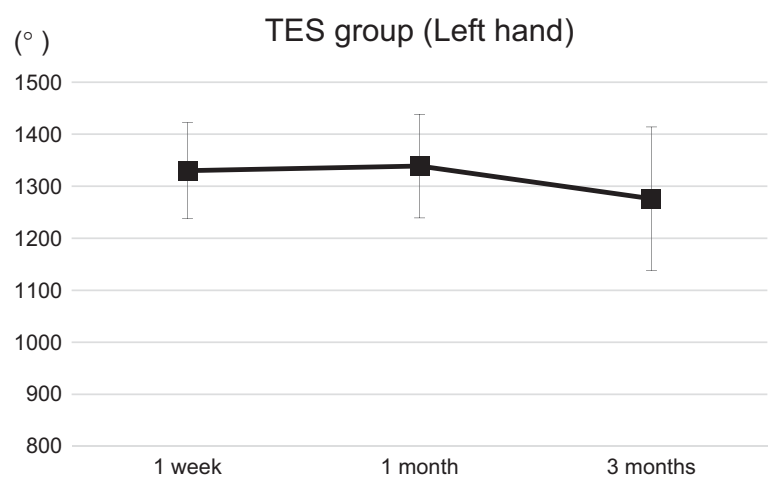

d

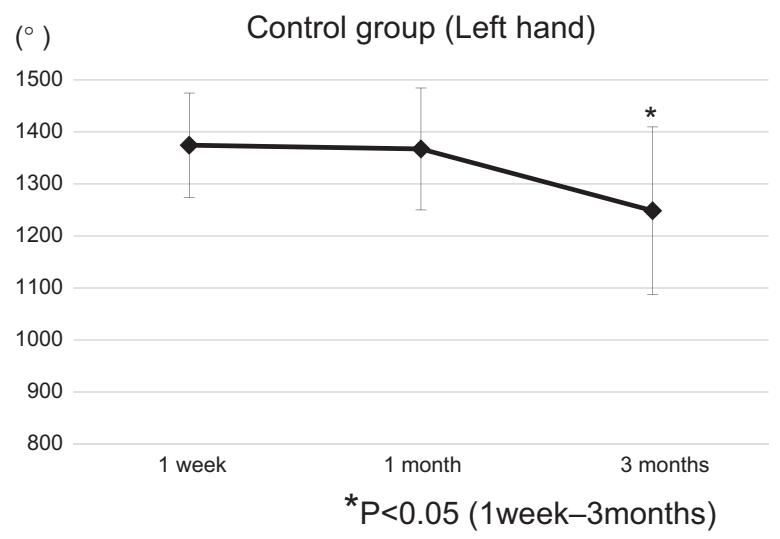

Figure 3 Comparison of total passive motion between 1 week and 3 months after injury. No significant differences were found in the TES group of both right hand (a) and left hand (b), whereas, significant decreases were found in control groups of both right hand (c) and left hand (d) at 3 months. Line plots were expressed as mean and s.d. 
After randomization, TES was initiated 1 week post injury. There were no significant between-group differences for TPM of the fingers, edema and upper-extremity motor scores at 1 week, 1 month and 3 months after injury, although TPM of the fingers tended to be lower in the control group (Table 2; Figure 3).

\section{DISCUSSION}

Kapadia et al. ${ }^{7,8}$ previously added TES to conventional rehabilitation training for the treatment of patients with spinal cord injury-induced paresis in the subacute or chronic phase (ASIA impairment scale B-D). They compared the ADL scores of patients receiving conventional rehabilitation training alone with those receiving conventional rehabilitation training and TES, and reported that TES had a beneficial effect. However, to our knowledge, there is no previous report of any randomized control study conducted which was commenced at the acute phase, and there is no evidence of the effect of the aforementioned treatment; therefore, we carried out a controlled study starting at 1 week after injury, that is, in the acute phase.

First, findings pertaining to the mean value of the TPM of the fingers showedno significant difference between the groups. We could not demonstrate the significant effect of TES on ROM in this clinical trial. On the other hand, when we look at the serial changes in the control group, the decrease of ROM of fingers was likely to occur at 3 months. Moriyama et al. ${ }^{13}$ previously stated that in joint contractures associated with spinal cord injury; moreover, both the muscles and the articular structures contributed to contracture development. It is possible that adding TES with muscle contraction to the conventional training might be more useful than passive ROM training alone for the prevention of contractures.

In addition, reports on the effects of electric stimulation in the treatment of edema are few, and Ralston et al. ${ }^{14}$ also reported the use of FES for the treatment of lower-limb edema, but they were unable to prove the beneficial effects of the treatment. When TES is performed, the articular motions triggered by electric stimulation are estimated to improve the pumping action of the muscles and to cause changes in blood flow volume, and this may potentially reduce edema; however, we were also unable to demonstrate the curative effect of TES on edema.

Findings pertaining to the upper-extremity motor scores showed that the upper-extremity motor score improved significantly over time by 3 months in both groups. Our study showed a similar recovery process to the results of previous studies; ${ }^{15,16}$ however, we were unable to demonstrate that TES itself was responsible for the recovery of the muscles.

In our study, a significant improvement in the upper-extremity motor scores was found at 3 months after the injury; however, in the control group, significant decrease of ROM of the fingers occurred at 3 months after injury. This suggested the importance of preventing decrease of ROM by carrying out rehabilitation throughout the acute phase, that is, until 3 months after injury.

The limitations to this study must be acknowledged. First, the sample size of this study was small. Large numbers of patients with cervical spinal cord injury should be evaluated in the future to strengthen the validity of our results and resolve this limitation. In addition, we did not blind assessors. Although we measured fairly without bias in each patient, blinded-endpoint trial (that is, blinding the assessors) would strengthen the results of this study.

An intervention involving rehabilitation starting in the acute phase is essential for preventing complications. Orthotic TES, which is safe, ${ }^{17}$ can be started in the acute phase. Our study did not provide definitive evidence that it prevents decrease in ROM in the rehabilitation of cervical spinal cord injuries, but it does provide initial data which future studies could build upon. In addition, it provides data which may be useful for meta-analyses. In future, ascertaining the indications of TES and determining the optimal training time ${ }^{18}$ will be necessary in order to offer more efficient rehabilitation.

\section{CONCLUSION}

This study prospectively examined the therapeutic efficacy of TES in patients with paresis associated with acute cervical spinal cord injuries, who were admitted within 1 week after injury. The observed decrease in ROM, which tend to be less in the TES group, over the 3 months that follow injury, that is, throughout a period of significant improvement of the paralysis, suggests the importance of rehabilitation for the prevention of decrease of ROM during the acute phase.

\section{CONFLICT OF INTEREST}

The authors declare no conflict of interest.

\section{ACKNOWLEDGEMENTS}

Works of TH and TM have been funded by the Grant-in-aid for Scientific Research (C) from The Japanese Ministry of Education, Culture, Sports, Science and Technology. This study reported here was supported in part by a medical research grant on traffic accident from The General Insurance Association of Japan. This study protocol was approved by the Institutional Review Board of Spinal Injuries Center. This trial was registered retrospectively with University hospital Medical Information Network (UMIN). The registration number was UMIN000026632.

1 Harvey LA, Herbert RD. Muscle stretching for treatment and prevention of contracture in people with spinal cord injury. Spinal Cord 2002; 40: 1-9.

2 Kapadia NM, Zivanovic V, Furlan JC, Craven BC, McGillivray C, Popovic MR. Functional electrical stimulation therapy for grasping in traumatic incomplete spinal cord injury: randomized control trial. Artif Organs 2011; 35: 212-216.

3 Alon G, McBride K. Persons with C5 or C6 tetraplegia achieve selected functional gains using a neuroprosthesis. Arch Phys Med Rehabil 2003; 84: 119-124.

4 Popovic MR, Kapadia N, Zivanovic V, Furlan JC, Craven BC, McGillivray C. Functiona electrical stimulation therapy of voluntary grasping versus only conventional rehabilitation for patients with subacute incomplete tetraplegia: A randomized clinical trial. Neurorehabil Neural Repair 2011; 25: 433-442.

5 Popovic MR, Popovic DB, Keller T. Neuroprostheses for grasping. Neurol Res 2002; 24 $443-452$.

6 Popovic MR, Curt A, Keller T, Dietz V. Functional electrical stimulation for grasping and walking: Indications and limitations. Spinal Cord 2001; 39: 403-412.

7 Kapadia NM, Bagher S, Popovic MR. Influence of different rehabilitation therapy models on patient outcomes: hand function therapy in individuals with incomplete $\mathrm{SCl}$. J Spinal Cord Med 2014; 37: 734-743.

8 Kapadia N, Zivanovic V, Popovic MR. Restoring voluntary grasping function in individuals with incomplete chronic spinal cord injury: pilot study. Top Spinal Cord Inj Rehabil 2013; 19: 279-287.

9 Frankel HL, Hancock DO, Hyslop G, Melzak J, Michaelis LS, Ungar GH et al. The value of postural reduction in the initial management of closed injuries of the spine with paraplegia and tetraplegia. Paraplegia 1969; 7: 179-192.

10 Zancolli E. Surgery for the quadriplegic hand with active, strong wrist extension preserved. A study of 97 cases. Clin Orthop Relat Res 1975; 112: 101-113.

11 Yamazaki H, Kato H, Uchiyama S, Ohmoto H, Minami A. Results of tenolysis for flexor tendon adhesion after phalangeal fracture. J Hand Surg Eur 2008; 33 557-560

12 Kirshblum SC, Burns SP, Biering-Sorensen F, Donovan W, Graves DE, Jha A et al. International standards for neurological classification of spinal cord injury. J Spinal Cord Med 2011; 34: 535-546.

13 Moriyama H, Yoshimura O, Sunahori H, Tobimatsu Y. Comparison of muscular and articular factors in the progression of contractures after spinal cord injury in rats. Spinal Cord 2006; 44: 174-181.

14 Ralston KE, Harvey L, Batty J, Bonsan LB, Ben M, Cusmiani R et al. Functional electrical stimulation cycling has no clear effect on urine output, lower limb swelling, and spasticity in people with spinal cord injury: a randomised cross-over trial. J Physiother 2013; 59: 237-243.

15 Fawcett JW, Curt A, Steeves JD. Guidelines for the conduct of clinical trials for spinal cord injury as developed by the ICCP panel: spontaneous recovery after spinal cord 
injury and statistical power needed for therapeutic clinical trials. Spinal Cord 2007; 45: 190-205.

16 Burns AS, Ditunno JF. Establishing prognosis and maximizing functional outcomes after spinal cord injury: a review of current and future directions in rehabilitation management. Spine (Phila Pa 1976) 2001; 26: S137-S145.
17 Peckham PH, Knutson JS. Functional electrical stimulation for neuromuscular application. Annu Rev Biomed Eng 2005; 7: 327-360.

18 Popovic MR, Thrasher TA, Adams ME, Takes V, Zivanovic V, Tonack MI. Functional electrical therapy: retraining grasping in spinal cord injury. Spinal Cord 2006; 44: 143-151. 